\title{
Modernidad, riesgo y la cultura de la prevención: una reflexión desde la
}

\section{intervención de Trabajo Social}

\author{
Modernity, risk and the culture of prevention: a reflection from the Social Work intervention \\ Francisco Omar Peña Guajardo ${ }^{a}$, Silvia María González Santos ${ }^{b}$
}

\begin{abstract}
:
This research article carries out an analysis in the context of the risk society and the intervention that Social Work has, for the development of an agenda that addresses the problems generated by environmental risks, it is worth mentioning that the elements are recovered theorists that refer to a reflective modernity, originated by the environmental technological crisis. Social Work, having the possibility of intervening for risk prevention, is an agent in the transformation of social relations, in the face of a vulnerable and devastating process for the globalization of the 21 st century.
\end{abstract}

\section{Keywords:}

Modernity, Risk, Social Work, Culture of Prevention, Environmental risk.

\section{Resumen:}

El presente artículo de investigación, realiza un análisis en el contexto de la sociedad del riesgo y la intervención que tiene Trabajo Social, para el desarrollo de una agenda que atienda a los problemas generados por los riesgos ambientales, cabe mencionar, que se recuperan los elementos teóricos que se refieren a una modernidad reflexiva, originada por la crisis tecnológica ambiental. Trabajo Social al tener la posibilidad de intervenir para la prevención de riesgos, es un agente en la transformación de las relaciones sociales, frente a un proceso vulnerable y de desolación para la globalización del siglo XXI.

\section{Palabras Clave:}

Modernidad, Riesgo, Trabajo Social, Cultura de la Prevención, Riesgo ambiental

\section{Introducción}

El objeto de estudio de las Ciencias Sociales, hoy en día, se constituye en contextos de emergencia. Los riesgos ambientales, representan un tema de mayor interés, al manifestar diversos factores que influyen en la falta de participación e interés por parte de los individuos, hacia los temas de prevención. Los daños y desastres tecnológicos ocasionados por los fenómenos naturales y el cambio climático son cada vez más predominantes en una sociedad del riesgo.

El presente trabajo de investigación aborda, un análisis sobre la cultura de prevención en situaciones de riesgos ambientales, desde la perspectiva de las Ciencias Sociales, que atiende las funciones y la importancia de la disciplina de Trabajo Social en el área emergente. Para cumplir con el objetivo de la investigación, se realiza una discusión desde la Teoría Social Contemporánea, que da una explicación relacionada a las consecuencias no deseadas, generadas por la pauperización ind ustrial y posteriormente tecnológica, en la modernidad, al ser parte de este campo contemporáneo, las teorías y enfoques del riesgo, principalmente, los de Ulrich Beck y Anthony Giddens, así mismo, se retomarán la propuesta hacia el fortalecimiento de una cultura de prevención en situaciones de riesgos ambientales, por parte del profesional de Trabajo Social, en el área emergente, al ser los desastres naturales, una causa de diversas problemáticas en las que el colectivo social se encuentra afectado, por lo que es, importante la postura del Trabajador Social como un agente social, en los procesos de intervención y transformación de una sociedad segregada,

\footnotetext{
a Autor de Correspondencia, Universidad Autónoma del Estado de Hidalgo, Escuela Superior de Tizayuca (ESTi). https://orcid.org/00000001-5582-9148, Email: francisco_pena@uaeh.edu.mx

b Universidad Autónoma del Estado de Hidalgo, Instituto de Ciencias Sociales y Humanidades (ICSHu). https://orcid.org/0000-0001-78782119, Email: smgsc95 @gmail.com
} 
desigual y vulnerable, como es la del riesgo, que a través de una crisis a nivel tecnológico y ambiental, manifiesta las consecuencias inesperadas de una globalización en decadencia.

\section{La sociedad del riesgo en la propuesta de Ulrich Beck}

El quehacer de Trabajo Social, es considerado diverso, puesto que existe de forma multidimensional su objeto de estudio, al ser los problemas y las necesidades sociales, por lo que, el área emergente en el que este interviene, juega también, un papel importante.

Una de las teorías, que permiten explicar el trabajo de investigación sobre la cultura de prevención y los riesgos ambientales desde la disciplina de Trabajo Social, en el campo de las Ciencias Sociales, es la obra del sociólogo Ulrich Beck La sociedad del riesgo escrita en la década de 1980, al explicar los efectos colaterales, provocados por la catástrofe de Chernóbil, considerada, como una de las catástrofes nucleares más impactantes, que ha sufrido Europa en el último siglo. Chernóbil era una de las centrales nucleares más importantes del mundo, el accidente nuclear fue consecuencia de un bajo nivel de seguridad.

La propuesta teórica del sociólogo Ulrich Beck, se centra en discutir, el tránsito de la modernidad industrial hacia una sociedad del riesgo, a través de una transformación producida por la confrontación de la modernidad, ante las consecuencias no deseadas en las propias acciones de los individuos (Beck, 1986).

El argumento básico de la teoría social en la sociedad del riesgo, es que, en el seno de la modernidad, se produce un proceso de cambio social y la industrialización, tiene una transición hacia la producción de mayores riesgos.

Tomando en cuenta la postura de Ulrich Beck, es preciso mencionar, que este analiza una realidad sin control o reflexión, que se da a partir de la industrialización y subsecuentemente, con el inadecuado uso de la ciencia y la tecnología, se producen daños de tipo ambiental, y el ser humano es el responsable de los riesgos antropogénicos, esta situación ha impactado con mayor frecuencia en la actualidad, específicamente en los temas del núcleo político, y no producir un tipo de impacto social al no generarse una preocupación, en tanto a temas de gestión del riesgo a desastres, seguridad, previsión y prevención de los riegos ambientales. El proceso modernizador, sigue en constante movimiento, la industrialización y el desarrollo de la tecnología están creando una nueva forma de organización social como lo menciona Ulrich Beck: “[...] el desarrollo industrial no regulado por el sistema político produce riesgos de una nueva magnitud: son incalculables, imprevisibles e incontrolables por la sociedad actual" (Citado por Huerta, 2011, p.1).

Por lo tanto, Ulrich Beck, considera que la sociedad del riesgo, implica una serie de cambios que se presentan en la realidad

\footnotetext{
*Acorde a los planteamientos del Sociólogo A. Giddens el concepto de lego es un concepto impreciso el cual hace una interpretación y compresión del mundo de diferentes formas a la del saber científico. Para profundizaren la
}

actual y que, a su vez, esos cambios invitan a reflexionar de manera crítica (Citado por Korstanje) los siguientes puntos:

a) El pasaje de una sociedad de clases a una sociedad de riesgos (según U. Beck, los riesgos alcanzan a todos puesto que todas las clases sociales se encuentran expuestas al riesgo).

b) El pasaje de una sociedad estamental, de identidades fijas sostenidas en la etnia, la religión, el trabajo, a una sociedad de individuación cada vez mayor, en la que las personas construyen sus trayectorias en forma reflexiva, escogiendo sus trabajos, sus parejas, sus modos de vivir.

c) El cambio del estatuto de la ciencia, desde una situación de monopolio del saber, hacia un escenario en el, que las opiniones de expertos compiten con el saber lego ${ }^{*}$ y con la racionalidad social (2010).

Se comprende, con base en las aportaciones de esta teoría, que la sociedad actual en general, se encuentra en constante riesgo, frente a amenazas que afectan de manera global, sin distinción de clases ni jerarquías. Los riesgos son generados por una modernización acelerada, que tienen en su mayoría un carácter antrópico, pues es el individuo quien, con el desarrollo de la técnica y de la ciencia moderna, así como, a través del crecimiento económico, crea los riesgos, y a su vez es carente de prevención, ante los fenómenos que pueden ocurrir.

Cabe mencionar, que el riesgo se presenta, no solo en el entorno, donde la vida natural se desarrolla, sino también, tiene un impacto en los sistemas económicos y de organización social.

Ulrich Beck reflexiona, el proceso de cambio de la modernidad industrial a la sociedad del riesgo, donde surge una nueva identidad de la sociedad caracterizada por la individualización humana (Dimuro, 2008). El individuo busca intereses, que atienden a responder a un tipo de racionalidad instrumental, además tiene un impacto en el aspecto del riesgo, y genera estrategias de prevención ante mayores riesgos imprevisibles e incontrolables.

Por lo tanto, en el análisis que realiza Ulrich Beck, el individuo realiza una reflexión de los riesgos, a los que está expuesto y a reformular sus prácticas sociales, que beneficien al colectivo social. También, se establece un término relevante al de Modernidad Reflexiva, como concepto esencial al de la obra de Ulrich Beck. La modernidad reflexiva, es algo nuevo que incorpora y desincorpora la tradición. Es una destrucción creativa como Beck lo menciona: [...] donde un tipo de modernización destruye otro y le modifica" (citado por Dimuro, 2008). Es importante mencionar, que todos los esfuerzos de definición se concentran en la figura del individuo. El individuo entiende y comprende la realidad social, y están condicionados a los más variados tipos de riesgo, los cuales tienen un alcance personal y global (Beriain, 1996).

Se debe entender en la teoría de Ulrich Beck, que el proceso modernizador implica diferentes factores, como el desarrollo de la ciencia y la tecnología, que ha llevado a la sociedad a generar dependencia, y descontrol. Por otra parte, en el ámbito

obra de Giddens, véase en: Giddens, A. (1993). Consecuencias de la modernidad, Alianza Editorial, Madrid. 
ambiental, tiene un impacto grave de repercusiones. Mientras la sociedad, no tome las decisiones y la reflexión adecuada y sea expuesta ante los fenómenos, que puedan ocurrir, el tema de la seguridad, seguirá puesta en riesgo y a través del tiempo se volverá aún más riesgosa. Finalmente, como lo expone U. Beck: "[...] la modernización reflexiva no tiende a la autodestrucción, sino a la autotransformación de los fundamentos de la modernización industrial" (Citado por Beriain, 1996, p. 223).

La postura que se considera, ante esta teoría, pese a que Ulrich Beck, aborda el riesgo desde su experiencia y en un contexto occidental, donde surgió una catástrofe de tipo nuclear, también, se plantean en sus aportaciones, una realidad que representa lo que se vive actualmente en diversos lugares del mundo, es decir, la forma en que la modernidad ha llevado a la socied ad, en convertirse en una sociedad del riesgo, al tener una influencia los daños ocasionados por los diversos factores económicos, sociales y culturales, etc., en un sistema capitalista global de producción.

\section{Aportaciones desde la modernidad reflexiva en la Sociología de Anthony Giddens: de una cultura del riesgo a una cultura de la prevención}

La modernidad reflexiva en la perspectiva del sociólogo Anthony Giddens, la define al relacionarla directamente con el riesgo social (Giddens, 1994). El teórico menciona, que las sociedades industriales, han producido consecuencias no previstas, que han transitado a través de riesgos patentes, ante los diferentes avances industriales y tecnológicos.

La vida social moderna, presenta un nuevo orden político social, y la modernización, ha generado un proceso de industrialización de gran importancia, en el que la ciencia, rompe con los esquemas tradicionales, concibiendo la relación del individuo y la tecnología, dado que los modos de vida y organización social a partir del siglo XVIII, han generado consecuencias que repercuten a nivel mundial, por lo que, se debe mostrar una nueva mirada sobre la naturaleza en la modernidad, que por ciertas razones se considera precariamente comprendida por las Ciencias Sociales.

Por su parte, Anthony Giddens señala categorías, que explican la modernidad desde una postura sociológica, presentado algunos conceptos como el de Discontinuidad de la Modernidad, al ser un proceso completamente diferente al de las sociedades tradicionales, caracterizado de un cambio y un constante movimiento, así como, la interconexión, enfocada a la comunicación entre diferentes regiones del mundo y el uso de la tecnología de forma general, en las instituciones sociales.

Del mismo modo el autor, hace referencia a las aportaciones de los teóricos clásicos (Marx, Durkheim y Weber), quienes vieron la "[...] era moderna como una era agitada. Pero ambos, pensaron que las beneficiosas posibilidades abiertas por la era moderna pesarían más que sus características negativas" (Giddens, 1994, p. 20), que no vislumbraron el enorme potencial de destrucción, que tendría la modernidad hacia el medio ambiente.

También, el autor trata de indicar, que anteriormente el individuo no se preocupó por lo que la naturaleza podía hacer, tomando en cuenta: terremotos, inundaciones, epidemias, malas cosechas y demás:

\begin{abstract}
En algún punto, ubicado más o menos en los últimos cincuenta años, dejamos de preocuparnos, tanto de lo que la naturaleza podría hacernos y comenzamos a preocuparnos más de lo que nosotros le hemos hecho. Esta transición, define uno de los principales puntos de entrada de la sociedad de riesgo. Es una sociedad que vive después de la naturaleza (Citado en Dettmer, 2002, p. 45)
\end{abstract}

A partir de ello, A. Giddens en sus aportaciones, discute acerca del tiempo y el espacio, al mencionar, que en las culturas premodernas, el tiempo estuvo conectado al espacio, puesto que, nadie podía saber la hora del día, sin hacer referencia a otros indicadores socio-espaciales. El invento del reloj mecánico y su difusión en los miembros de la población, fueron de crucial importancia en la separación del tiempo y el espacio:

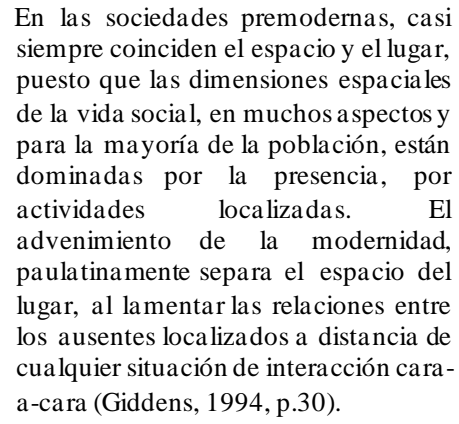

El aspecto ambiental, se ve afectado en el tiempo y el espacio, debido a que la transformación y los cambios en la naturaleza, adquieren nuevos riesgos, un ejemplo, es el cambio climático, que en las sociedades precedentes era más previsible, a partir del proceso modernizador, el cambio climático se ve afectado, por lo que, la sociedad actual no prevé los daños que esto y otras consecuencias puedan ocasionar.

El control del espacio, permite reconocer los contextos locales, a través de un impacto global. Por otra parte, el proceso de desmembramiento (desanclaje, desvinculación), implica dos tipos de situaciones: “[...] a) un profundo proceso de reorganización del tiempo y el espacio (mecanismos que liberan a las relaciones sociales de la influencia de los emplazamientos locales recombinándolas a través de amplias distancias espaciotemporales) y b) el vaciamiento del contenido tradicional (acabar con la costumbre de los contextos locales de acción)" (Giddens, 1993, p. 32).

Es pertinente mencionar, que el término de desmembramiento y/o desanclaje, tiene un significado enfocado a separar las relaciones sociales de sus contextos locales de interacción y reestructurarlas, lo cual permite, una nueva organización social, en la que se promueva la prevención desde una mirada consciente y haciendo uso adecuado de las herramientas de la modernidad, como lo es la tecnología, entendiendo que todos los mecanismos de desanclaje, así sean señales simbólicas y/o sistemas expertos, descansan sobre la noción de fiabilidad tanto que, la fiabilidad va implicada, de manera fundamental, en las instituciones de la modernidad; pero esa fiabilidad no se confiere a individuos sino a capacidad es abstractas. 
Por ejemplo, desde una perspectiva, que está vinculada con el dinero, cualquiera, que utilice los símbolos monetarios, lo hace asumiendo que los otros, a los que nunca ve, respetarán su valor, de la misma manera desde el aspecto ambiental si se introducen símbolos hacia una cultura de prevención, de manera abstracta en el espacio, existiría la fiabilidad de adquirir nuevos valores, que generen un control de desastres de los riesgos ambientales.

A diferencia de Ulrich Beck, la postura que nos aporta el sociólogo Anthony Giddens es contraria, debido a que la modernidad y todo lo que esta conlleva, permite generar acciones para aminorar el riesgo, haciendo uso adecuado de la tecnología y la reflexión por parte de la sociedad, teniendo en cuenta, que el individuo en un proceso histórico, ha tomado nuevas formas de consciencia y acción, por lo que pueden tomar decisiones con mayor reflexión.

Las aportaciones de Anthony Giddens, permiten comprender, que la modernización y el uso de la tecnología, se deben de aplicar de manera moderada y adecuada, pues el individuo se enfrenta a una novedosa serie de riesgos y posibilidades.

\section{Cultura de la Prevención e Intervención de Trabajo Social}

La prevención es un término muy común, siempre que pensamos en tratar de actuar antes de que algo no deseado suceda, estamos pensando en clave preventiva.

El profesional en Trabajo Social, a través de su matriz metodológica estructurada por un proceso operativo que está presente en todas las formas de intervención del Trabajo Social (Galena, 2005), al conocer y tener un acercamiento más cercano de la realidad social, genera planes y proyectos, y/o en su caso se hace necesario un nuevo modelo de Trabajo Social y un nuevo perfil profesional entendiendo que: "El ejercicio profesional presenta una diversidad de formas de intervención" (142 p.), pues el objeto de estudio es multidimensional, al ser diverso y cambiante.

Se debe de fundamentar por enfoques y teorías que expliquen la realidad social con una actuación de objetividad, hacia ellos deberían ir dirigidos muchos de los esfuerzos desde distintos campos y ámbitos de influencia de los trabajadores sociales en una era de marcado individualismo al que no somos ajenos, es decir, en ocasiones Trabajo Social se encuentra limitado en sus funciones por diversos factores, como el estigma de la profesión, las instituciones en las que este profesional se desarrolla, entre otros.

El profesional de Trabajo Social, tiene la capacidad de inferir en la formación de una cultura de prevención en los riesgos ambientales. Prevenir no es sólo evitar que algo suceda, para de este modo ahorrar recursos en momentos de crisis, y/o evitar gastos mayores.

Prevención es apostar por un futuro mejor para muchas personas, para mejorar el bienestar y la calidad de vida de muchas otras. Prevención significa planificar desde abajo, sin urgencias, manteniendo los programas a medio y largo plazo, independientemente de los distintos grupos en el poder.

En prevención social, más que hablarse de individuos en riesgo, sino de contextos de riesgos, tratando de favorecer más los indicadores de protección, que la identificación de los indicadores de riesgo. Del mismo modo, no debería remarcarse solamente las dificultades, también, las oportunidades que ofrece para la prevención-promoción cualquiera de los niveles de un modelo ecológico*

El profesional en Trabajo Social, puede aplicar un modelo de corte preventivo para los tres niveles de intervención: caso, grupo y comunidad, superador del asistencialismo; reparador, educativo, promocional y participativo; creador y mantenedor de tejido social y en contacto e interrelación con otros servicios, organizaciones e instituciones presentes en su ámbito de referencia (Raya, 2005).

La prevención es una gran oportunidad para Trabajo Social que, por su proximidad a los ciudadanos y a las situaciones carenciales cotidianas y/o estructurales, disponen de un conocimiento privilegiado para, con la participación de la trama social, de las personas, grupos y colectivos, construir nuevos escenarios de intervención, pasando de una atención residual (con los efectos) y mayoritariamente individual-familiar, a otro tipo de acciones más colectivas, que busquen tanto la prevención de los riesgos, como la educación y promoción de las personas, grupos y colectivos en una red de relaciones que potencialicen la autonomía y la responsabilidad (Domínguez, 2001).

A partir de la propuesta en Trabajo Social, es necesario reflexionar sobre la necesidad de intervenir ante nuevos desafíos, e integrarlos a los pendientes de las agendas, sobre todo, por el contexto de globalización que marca actualmente los pasos de la sociedad moderna, en el que se siguen generando nuevas problemáticas y exacerbando las que existente.

\section{Conclusiones}

La propuesta de Trabajo Social en un contexto de modernidad reflexiva y/o sociedad del riesgo, apunta a reconocerse como uno de los principales agentes en la prevención de riesgos ambientales, en una crisis soslayada que se vive ante un cambio climático y el uso de la tecnología, estableciendo de la siguiente manera algunos puntos a considerar:

1.- Los argumentos teóricos nos permiten comprender que el tránsito de la modernidad, ha provocado una sociedad del riesgo, de forma relevante en el impacto ambiental, donde las propias acciones del ser humano como el uso inadecuado de la tecnología y la razón instrumental, han generado consecuencias no deseadas, y, además, tienen un carácter global.

2.- Las aportaciones de Ulrich Beck, permiten reflexionar una modernización reflexiva, al ser una característica de la transformación, en los actos del individuo ante los riesgos posibles.

enfoque comunitario. Departamento de Trabajo Socialy Servicios Socia les. Universidad de Alicante, pp. 135-168, permite una revision desde la prevención en Trabajo Social.

\footnotetext{
* Para una revision del modelo ecológico en Trabajo Social, véase documento de Domínguez, J. (2001). Actuaciones preventivas en contextos comunitarios. Una oportunidad, ¿necesidad? para el trabajo social con
} 
3.- Por otra parte, la perspectiva de Anthony Giddens, permite plantear que la ciencia, en su uso adecuado, y fiable, adquiere un tipo de control al tomar decisiones, en mayor reflexión, tomando en cuenta, las acciones de rutina y cotidianeidad de una política ambiental, que conozca e intervenga en los contextos locales, para su impacto en el ámbito global.

La modernidad, como la consideran los autores, tiene un lado positivo y negativo, pues al ser un proceso incontrolable, la sociedad se encuentra con mayor riesgo, por las consecuencias provocadas en la naturaleza, en relación de sus actos y la toma de decisiones, pues al suceder un desastre natural y/o antrópico, la intervención es carente, en otros casos la sociedad no posee elementos, que generen una cultura de prevención, debido a las desigualdades sociales presentadas en función de la pobreza. Existen diversos factores, que engloban la problemática del riesgo y que aquejan a gran parte de la sociedad, el problema, ahora, no es la conquista de la naturaleza, sino, evitar la destrucción y degradación de la misma.

4.- Finalmente, el Trabajador Social tiene que actuar como un Agente Social $^{*}$ de cambio en áreas emergentes, en situaciones de riesgos ambientales y de cultura de la prevención, al considerar con otros enfoques y/o perspectivas que permitan interpretar las prácticas sociales que parecen incomprensibles, irracionales y/o paradójicas, en la construcción de diagnósticos más precisos dotados de objetividad (Bermúdez, 2019), y su intervención a nivel social, constituya uno de los espacios a través de los cuales el ejercicio profesional, mantenga una de sus más claras expresiones.

\section{Referencias}

[1] Beck, U. (1986). La sociedad del riesgo, hacia una nueva modernidad Barcelona, España.Paidós.

[2] Beriain, J. (1996). Las consecuencias perversas de la modernidad, Barcelona, España. Antrophos.

[3] Bermúdez, C. (s/f). Intervención social desde el Trabajo Social: un campo de fuerzas en pugna. Disponible en: http://bibliotecadigital.univalle.edu.co/bitstream/10893/6316/4 Prospectiva\%2016\%2C\%202011-83 101\%20Intervenci\%C3\%B3n\%20social\%20desde\%20el\%20Tr abajo\%20Social.pdf.

[4] Dettmer, J. (2002). Educación y desastres: reflexiones sobre el caso de México. Revista Latinoamericana de Estudios Educativos. 32 (2), pp. 43-72.

[5] Beck, U. (1986). La sociedad del riesgo, hacia una nueva modernidad Barcelona, España. Paidós.

[6] Beriain, J. (1996). Las consecuencias perversas de la modernidad, Barcelona, España. Antrophos.

[7] Bermúdez, C. (s/f). Intervención social desde el Trabajo Social: un campo de fuerzas en pugna. Disponible en: http://bibliotecadigital.univalle.edu.co/bitstream/10893/6316/4 /Prospectiva\%2016\%2C\%202011-83

\footnotetext{
* Para profundizar más en la obra de Pierre Bourdieu y su concepto de Agente, se puede revisaren Fernández, J. (2003). Habitus y sentido práctico:
}

101\%20Intervenci\%C3\%B3n\%20social\%20desde\%20el\%20Tr abajo\%20Social.pdf.

[8] Dettmer, J. (2002). Educación y desa stres: reflexiones sobre el caso de México. Revista Latinoamericana de Estudios Educativos. 32 (2), pp. 43-72.

[9] Dimuro, G. (2008). Los ecosistemas como laboratorios la búsqueda de modos de vivir para una operatividad de la sostenibilidad. Universidad de Sevilla Escuela Técnica Superior de Arquitectura Máster Oficial en Ciudad y Arquitectura Sostenibles Especialidad Investiga ción, pp. 1-200.

[10] Domínguez, J. (2001). Actuaciones preventivas en contextos comunitarios. Una oportunidad, ¿necesidad?, para el trabajo social con enfoque comunitario. Departamento de Trabajo Social y Servicios Sociales. Universidad de Alicante, pp. 135168.

[11] Fernández, J. (2003). Habitus y sentido práctico: la recuperación del agente en la obra de Bourdieu. Cuadernos de Trabajo Social, 16, pp. 7-28.

[12] Galeana de la O, S. (2005). Campos de acción del trabajo social. En M. Sánchez Rosado (Coord.), Manual de Trabajo Social. (pp. 139-158). México: Plaza y Valdés.

[13] Giddens, A. (1993). Consecuencias de la modernidad, Alianza Editorial, Madrid.

(1994). Consecuencias de la modernidad. Alianza editorial. Madrid, España.

[14] Huerta, D. (2011). Ulrich Beck: "La sociedad del riesgo". Awareness`s blog. Obtenido de: https://davidhuerta.typepad.com/blog/2011/03/ulrich-beck-lasociedad-del-riesgo.html Blog

[15] Korstanje, M. (2010). Reseña de "La sociedad del riesgo: hacia una nueva modernidad" de Beck, Ulrich. Economía, Sociedady Territorio, 10 (32), pp. 275-281.

[16] Raya, E. (2005). Tema 3 Modelos de intervención en Trabajo Social Comunitario. Universidad de la Rioja. Disponible en: https://www.unirioja.es/dptos/dchs/archivos/TEMA3MODELO S.pdfCurso.

la recuperación del agente en la obra de Bourdieu. Cuadernos de Trabajo Social, 16, pp. 7-28. 